\title{
COVID-19 as a driver for change in audiovisual archives
}

Six observations from a virtual exchange between professionals

Maria Drabczyk, National Film Archive - Audiovisual Institute, Poland, Value, Use \& Copyright Commission FIAT/IFTA

Johan Oomen, Netherlands Institute for Sound and Vision, Organising Knowledge Committee IASA

FIAT IFTA liasa 


\section{Colophon}

Amsterdam \& Warsaw, March 2021

FIAT/IFTA

\section{Authors:}

Maria Drabczyk, National Film Archive - Audiovisual Institute, Poland, Value, Use \& Copyright Commission FIAT/IFTA Johan Oomen, Netherlands Institute for Sound and Vision, Organising Knowledge Committee IASA

\section{Contributors:}

Dale Grayson, Northbound TV, Value, Use \& Copyright Commission FIAT/IFTA

Louise Broch, DR, Value, Use \& Copyright Commission FIAT/IFTA

Montserrat Bailac, TV3, Value, Use \& Copyright Commission FIAT/IFTA

and all the participants of the virtual exchange and the respondents to the FIAT/IFTA 'Where are you on the Timeline' survey.

Graphic design: Magdalena Arażny, magdarysuje.pl

Proofreading: Arthur Barys

This report is published under a Creative Commons CC BY-SA 4.0 licence. 


\title{
Foreword
}

\section{"If you want to go fast, go alone. If you want to go far, go together."}

\author{
- African proverb
}

The audiovisual archiving community unites professionals located across the globe. Umbrella organisations, including the two that supported this research, FIAT/ IFTA and IASA, play a vital role in bringing the community together and facilitate the exchange of ideas and insights. The positive effects of belonging to a community cannot be overstated, especially during the period of great uncertainty caused by the COVID-19 pandemic. It required swift adaptation of working processes and exposed existing weaknesses involving connectivity, remote working, and economic dependencies. The community stepped up, not only providing much-needed practical advice, but also by offering moral support to peers facing similar challenges. The message that 'we are in this together' is empowering. The lively conversations that form the basis of the observations shared in this document are testament to the power of the archival community.

The past year of disruptions and adaptations has taught the community many lessons: about reaching out online, remote working, and, more generally, about its resilience in times of crisis. The global pandemic condensed a decade of digital transformation into a few months. This report captures the various ways in which the cultural heritage sector is adapting, not only to cope with the present, but also to flourish in the future. As vaccines become available - and though addressing the great disparity between countries remains a matter of utmost urgency - the community is starting to strategise about its long-term recovery. We hope that the observations shared here will be helpful in the future.

Discussing openly which approaches work and which do not is always difficult. We wish to deeply thank everyone who contributed to this work.

Maria Drabczyk Johan Oomen 


\section{Context and approach}

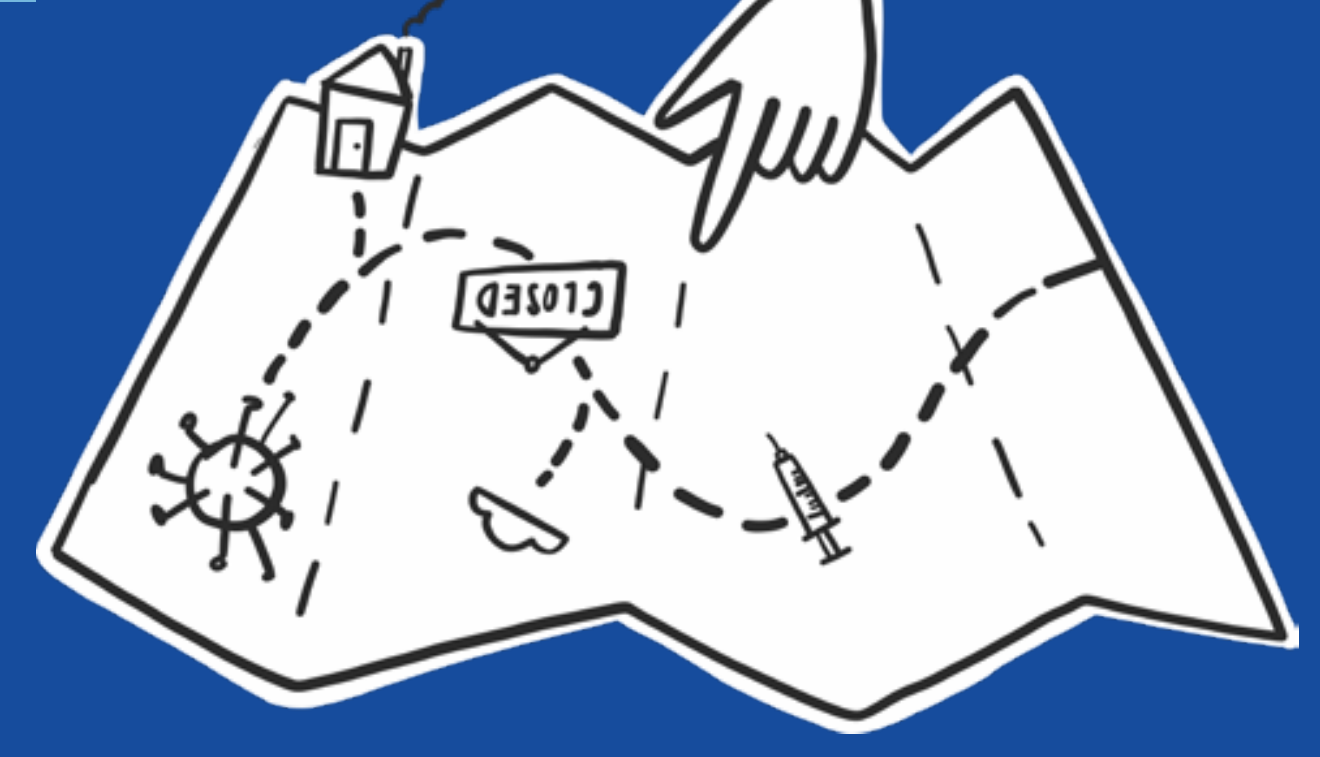




\title{
Turning Crisis
}

\section{into Opportunity?}

\author{
"To create a resilient and successful cultural sector, not one that stagnates and falls back \\ into the past, we need to create space for innovation and experiments; learn from each \\ other's failures and successes; and allow for playful and creative engagement with the \\ big ideas that will define the future."
}

\section{- Michael Edson and Jasper Visser}

Since the beginning of the coronavirus outbreak in early 2020, the cultural and creative industries have been forced to adapt quickly and reposition themselves in a dramatically different operational context. Most of the discussions and reports regarding the COVID-19 pandemic and its impact on the sector have focused on the negative consequences. But the emergency has also triggered unusual acceleration in the development of existing ideas and the creation of completely new ones with regard to online access, copyright, new creative ways to use collections, etc.

In the OECD report Culture shock: COVID-19 and the cultural and creative sectors, ${ }^{1}$ published in September 2020, we read that the titular industries were among the hardest hit by the pandemic. At the same time, the report states that the emergency created an opportunity for change in the field of digitalisation, paving the way for new online experiences and audience engagement.

'The massive digitalisation in recent months in the culture and creative sectors is clearly not temporary and could create new forms of experience and business models with market potential. There is an opportunity for a major innovation breakthrough in terms of the deployment of state-of-the-art technologies that allow "presence at a distance" (artificial intelligence, virtual and enriched reality, Internet of Things, etc.) to build a new "experience economy". New forms of digitally mediated, decentralised creative production allow for engagement with larger and wider communities, not only at the receiving end but also in terms of content production.'

Culture shock: COVID-19 and the cultural and creative sectors

Various heritage communities and associations, like Europeana, the Network of European Museum Organisations, and others, also used the momentum to map and evaluate the impact of the crisis on their professional communities, and made efforts to turn the challenges into valuable learning experiences, creating space for future change. 


\section{AV Community Perspective}

An approach similar to the ones listed above was adopted by the archival audiovisual community. In October 2020, the International Association of Sound and Audiovisual Archives (IASA) and the International Federation of Television Archives (FIAT/IFTA) invited their members to a joint virtual exchange.

The initiative was set up to allow the community to share their experienceto discuss the challenges and changes they had encountered at the strategic level and in their daily operational routines. The aim was to gather professionals from the global archival community and to discuss how positive changes could be identified and sustained, to share best practices and individual experiences, and to collaboratively think out the best ways forward. The underlying intent of these efforts was to explore how the emergency acted as driver for change, and especially to highlight the changes and adaptations that lead to long-lasting positive effects and increased resilience.
The core of the exchange was built around two online sessions held in early October 2020.

More than 20 audiovisual archival professionals from all over the globe (Figure 2 illustrates the geographic distribution) took part in the exchange directly, either by filling
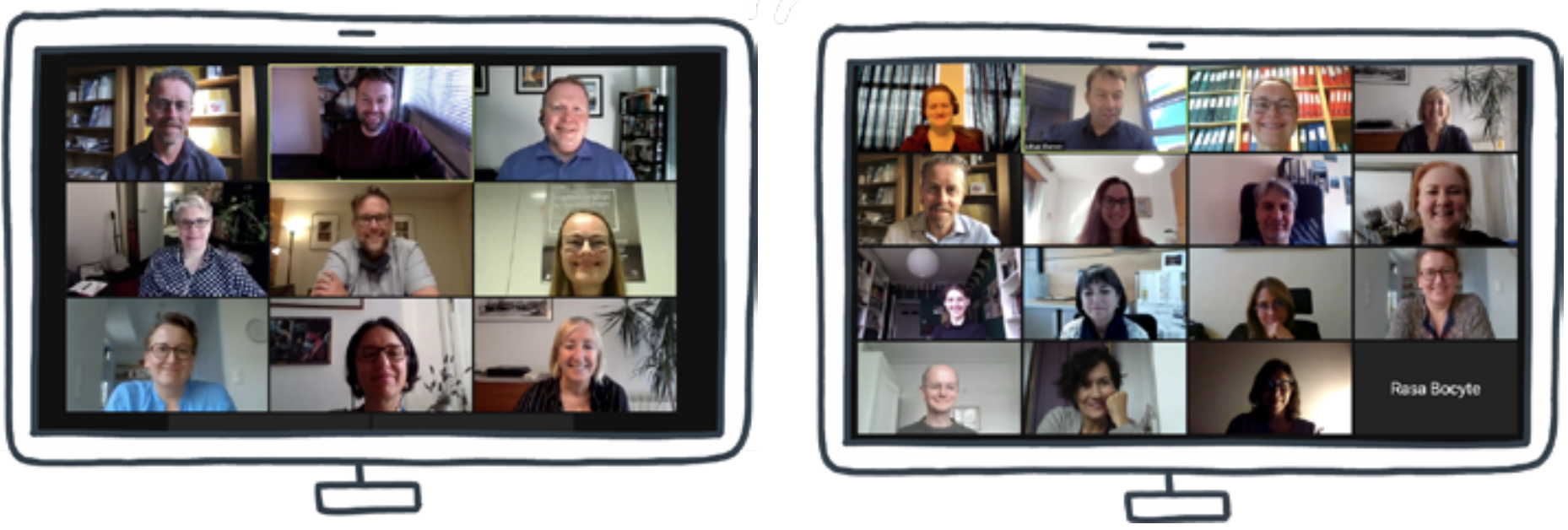

in the questionnaire, by participating in one of the online sessions, or by contributing to both.

To gather more data and to facilitate the online discussions, all registered participants were asked to fill in a pre-questionnaire
Figure 1: Participants attending the two sessions of the virtual exchange held online in October. 
about their personal observations on the topic. Additionally, to better map the situation in the global audiovisual archival community, targeted questions were added to the FIAT/IFTA 2020 edition of the annual 'Where are you on the Timeline'2 survey. This brought in the additional perspectives of 80 organisations from all over the world who completed the FIAT/IFTA survey (not included in Figure 2).

There's a considerable body of related research in the heritage sector. We refer readers to notable publications by the $O E C D{ }^{3}$
Europeana, ${ }^{4}$ UNESCO, ${ }^{5}$ the European Commission, ${ }^{6}$ and NEMO. ${ }^{7}$ These studies provide analyses across various heritage sectors, with a focus on the economy. They are a great companion to the observations provided in this FIAT/IFTA study.

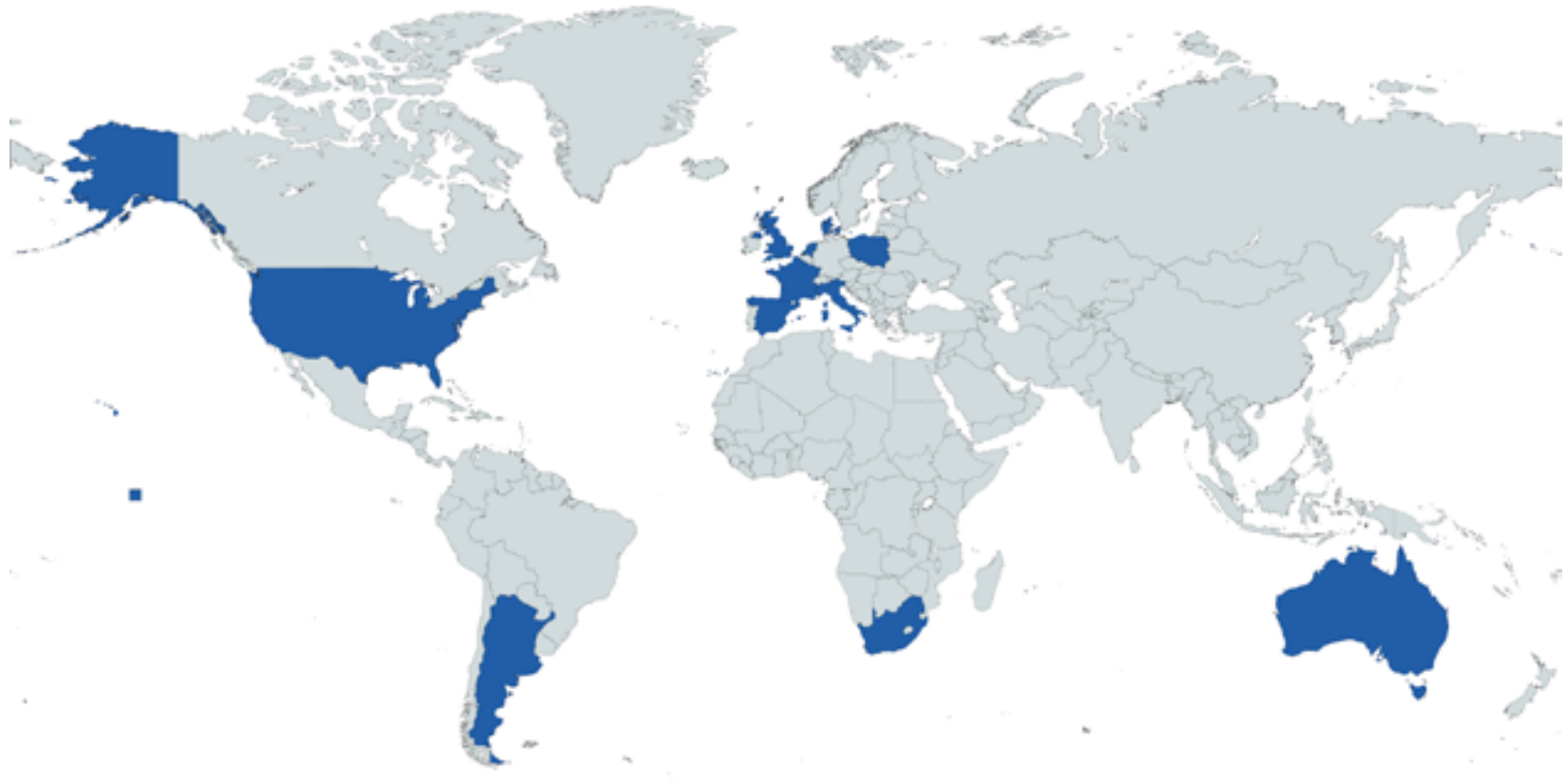

Figure 2: Geographic distribution of AV professionals who contributed to the exchange.
2 https://fiatifta.org/index.php/2020/09/18/fiat-ifta-where-a re-you-on-the-timeline-survey-2020

${ }^{3}$ http://www.oecd.org/coronavirus/policy-responses/culture-shock-covid-19-and-the-cultural-and-creative-sectors-08da9e0e/

${ }^{4}$ https://pro.europeana.eu/post/building-digital-capacity-sense-making-findings-and-outcomes

${ }^{5}$ https://en.unesco.org/news/launch-unesco-report-museums-around-world-face-covid-19

${ }^{6}$ https.//wwweuroparleuropaeu/thinktank/en/document. html? reference=IPOL STU(2021)652242

https://www.ne-mo.org/advocacy/our-advocacy-work/museums-during-covid-19.htm 


\section{Observations}

What did we, as an archival community, learn from this experience? Based on the virtual exchange and the results of the survey, we identified six key observations. We believe they must be addressed if the audiovisual archival community is to remain relevant, connected, and in sync with its stakeholders and audiences and their needs. Furthermore, these findings are relevant to the way archives operate as organisations in the time of crisis. 


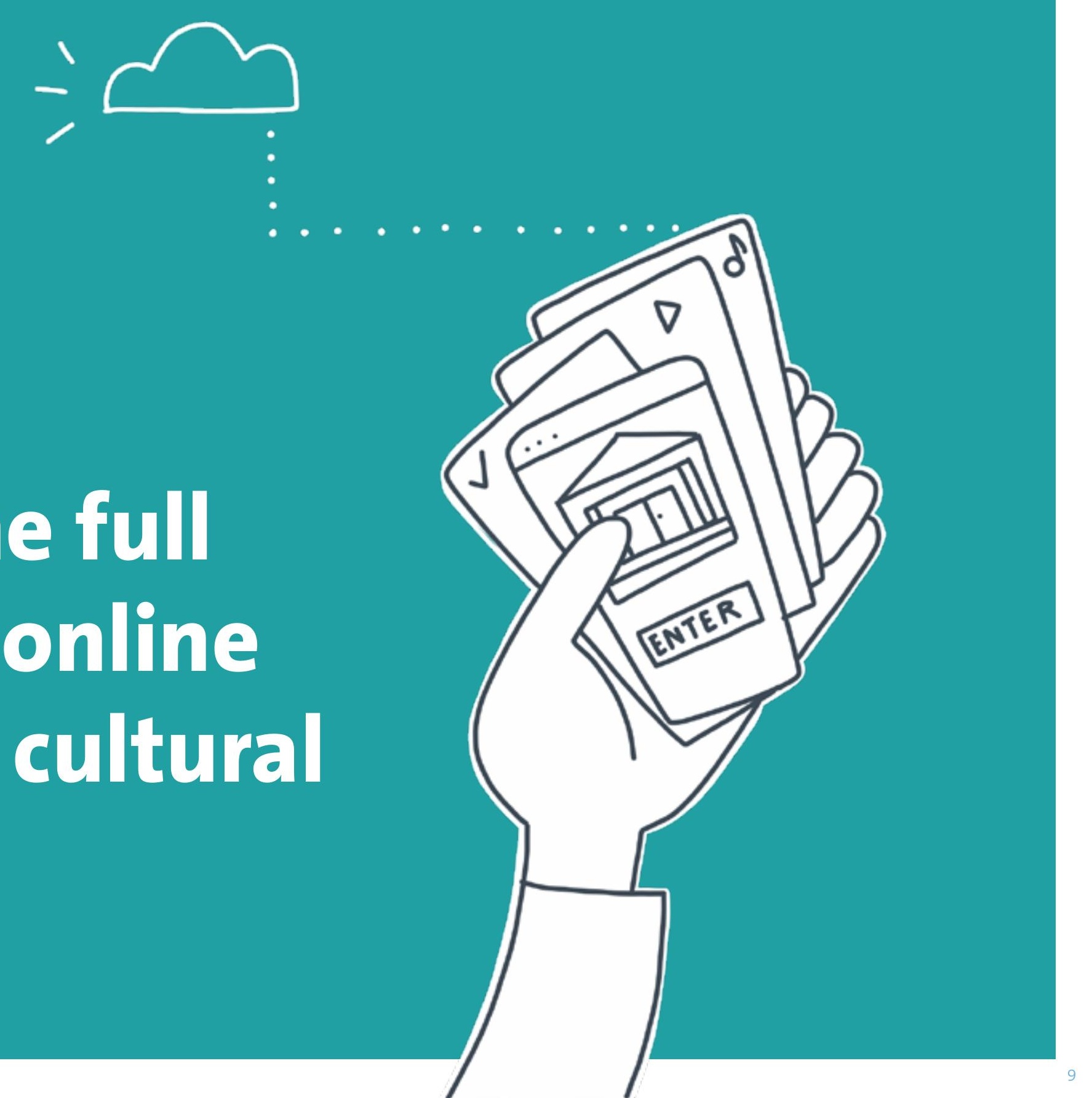


Over the past decade, investments were made to offer access to collections to specific user groups, through such channels as online catalogues, archive-based productions in radio and television, and theme-based digital learning platforms for schools.

With the emergence of social media, archives expanded their reach through third-party platforms ranging from YouTube to Facebook and Wikipedia. Audiovisual archives have, in short, always taken strides to align their offerings with user expectations and, at the same time, to investigate how the latest technological developments can offer new opportunities for access and exploration.

One trend is common across the globe: interest in archival content has been on the rise since the start of the pandemic. Especially now, with access to physical venues restricted, the importance of online access has become paramount. In the conversations, it became clear that archives are increasing their efforts to bring content online. Some institutions needed to play catch up as infrastructure wasn't yet in place, while others could expand their existing offering.

Many new opportunities are on the horizon: from using TikTok and Instagram, to reaching out to a younger demographic. Additionally, technologies like extended
Reality, recommender systems, and synthetic media can be explored as the basis for new ways of storytelling. The use of artificial intelligence (Al) is also giving rise to new forms of creativity. We learned of archives that had created chatbots on apps such as Messenger and Telegram, giving users personalised access to archival collections. $\mathrm{Al}$ is also helping to create interfaces that support exploration over searching, or what are known as generous interfaces.

It should be noted that this demand for more archival content isn't exclusive to the online domain: in our conversations, we also learned of growing demand for archival content disseminated via 'traditional' linear television and radio. In some countries, daytime television was expanded to provide more entertainment for people as a way to cope with being cooped up at home.

One debate that has gained more prominence over the past year involves the matter of which considerations should be taken into account when choosing between different content distribution solutions. Aside from the obvious factors (cost, usability, scale, quality of service), increasing consideration should be given to aligning technology with public values. Does the system track users? Who owns data created by the system? Are the algorithms open and accessible, so that we can trace bias and take corrective action? Are we comfortable showing online advertisements that collect user data on our portals? It is important for institutions to strategize which considerations are important for them, given their missions, as these choices will greatly affect successful technical implementation, the cost perspective, and rights of end-users.

On a more macro-economic level, entertainment, education, social interaction, commerce, and politics are being transferred to the virtual world. This process has accelerated considerably due to the pandemic. In the virtual world, a handful of digital-content platforms (Netflix, Amazon, Google, etc.) and social-media platforms (Facebook, Twitter, etc.) have enormous influence on the way information is distributed, and they exert pressure on public spaces on the internet. There is growing concern, within society and the heritage community, about the power wielded by these market-driven platforms. Lawmakers in different countries are considering which legal actions they will need to take to support pluriformity, openness, and privacy online. Audiovisual archives will also need to consider how they can contribute to an internet that is more friendly to public values. 
Providing access is always closely connected to managing copyright. In our conversations, it became apparent that some rights holders that have historically been resistant to online access have shifted their thinking, and have become more willing to consider the option. As before the pandemic, archives seek permissions from rights holders to provide access to use of archival content when possible. If rights status is unknown or unclear, standardised models (such as the online publication flowchart accessible via the FIAT/IFTA website ${ }^{8}$ ) are available to make a risk assessment. As seen in Figure 3, over half of the participants in the FIAT/ IFTA survey agree with the statement that free access should be prioritised over monetization of assets. A sizable proportion of the community has expressed a more neutral or negative position towards this statement. This could be traced to the fact that the financial valorisation of archival content (e.g. through footage and rights sales) remains an essential source of revenue for many broadcasters. Additionally, there may be reasons related to ethical or privacy considerations for not opening certain parts of broadcast archives to the (general) public.

In light of the COVID-19 crises it is important to aim for a greater reach with the public by offering free access to online content, rather than trying to increase revenues, for example through the sale of archival footage

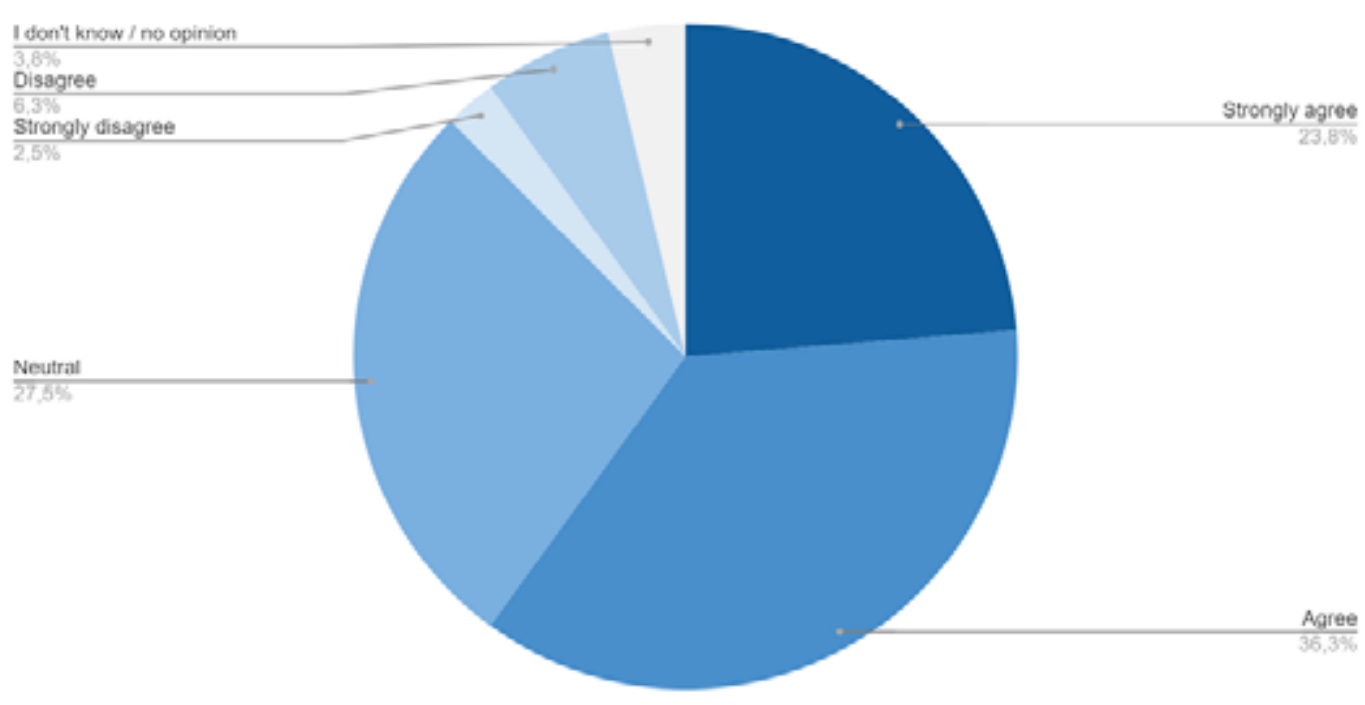

Figure 3: FIAT/IFTA Timeline survey - free access vs. monetization 


\section{observation 2: \\ Foster direct \\ engagement \\ with your \\ audience}

(2) 四 
The past year has clearly demonstrated the value of digital access to culture for global society, particularly in a time when most cultural institutions were shuttered for months on end. Many of the participants in the exchange emphasized that the availability of digital cultural content during the pandemic and the mere fact that many institutions had made their content available online contributed to the viewers' mental health and well-being. Online access to, and contact with, culture created a sense of belonging to a community, despite the temporary physical separation. It was also recognised that the time of isolation created space for a more personal interaction between the archive and its audiences. Archives were reaching out to their audiences by leveraging the full potential of social media (discussion groups, chats, communicators, etc.), through an immense increase in the number of online screenings followed by O\&A sessions with the audience, by organising online workshops and meetings, etc. The approach proved the value of personal context and personal cultural experience.

Furthermore, the enormous intensification of online activities, no longer limited to a specific geographic location, created a unique opportunity for the archives to not only rebuild their relationship with their existing audiences, but also to reach a new audience, one eager to vividly participate in online culture, to learn about the institution's offer, and sometimes to contribute creatively, as well, by taking part in crowdsourcing initiatives run by the archives (for instance, by sharing their private pandemic memoirs).

Some archives decided to take it a step further, inviting their audiences to use their collections creatively and become virtual curators

or commentators. Archives started to be more attentive to the voices of their users and began to recognise cultural experience as a co-creation between professionals and the public.

All of the changes in audience engagement listed above have pressured institutions to strengthen existing audience-oriented competences (soft skills) or acquire new ones, and to match them with the tools necessary to host, for example, a friendly webinar. Some archives have achieved this simply by improving on already implemented user engagement strategies. For others, it meant facing the unknown waters of online engagement and a new type of communication and interaction with audiences.

This new level of intensification of interaction with various stakeholders (media professionals, patrons, researchers, educators and students, the general public, etc.) has definitely challenged archives to better tailor their access offer to the needs and wishes of their audiences (old and new), improve their discoverability and user experience, and secure remote access to collections and catalogues for educators, researchers and creatives (see Figure 4).

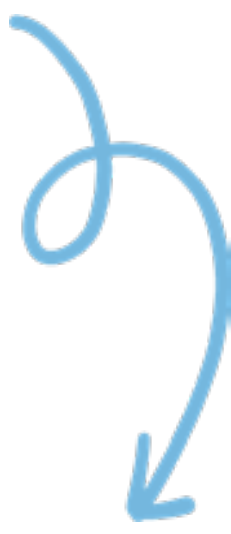


In light of the COVID-19 crises it is important to tailor the supply

of audiovisual archive content, such as education, instead of

a one-platform-serves-all approach

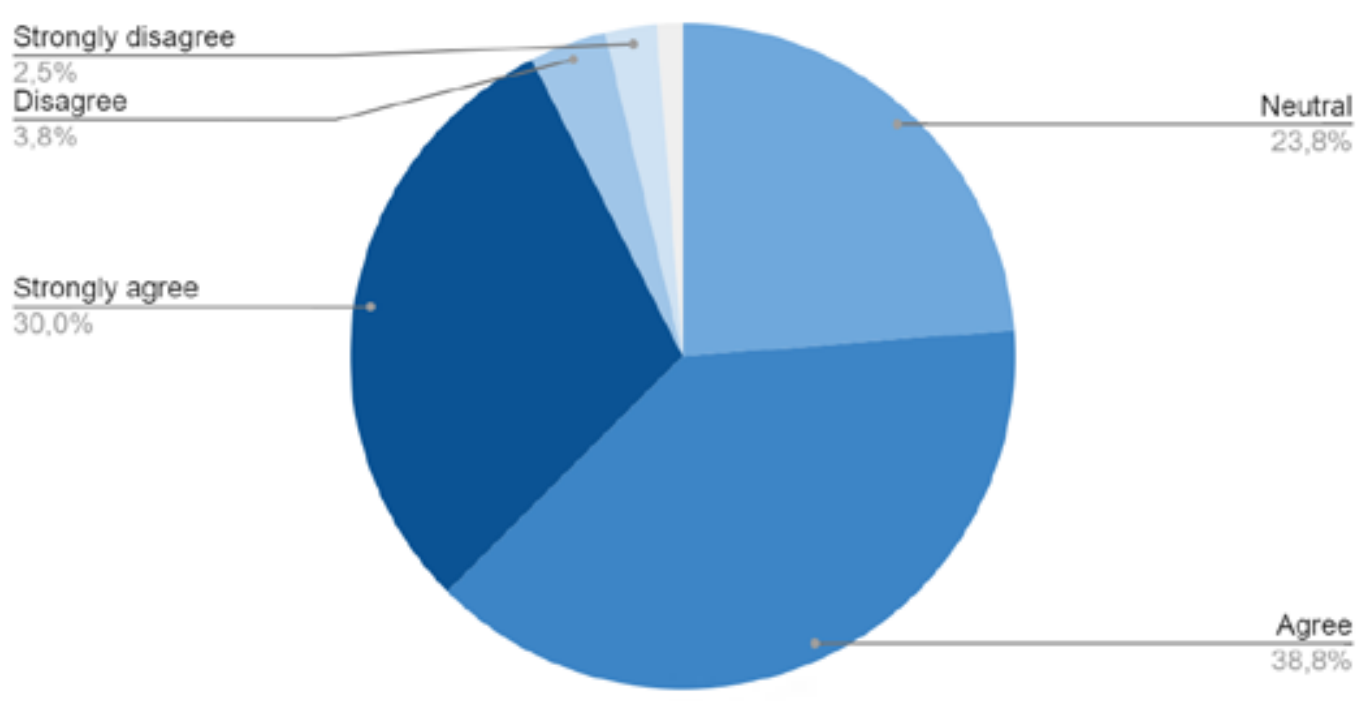

The pandemic has given the community time and the sometimes-needed push to rethink its relationship with its stakeholders, and to be more responsive to the sudden change in expectations as the world moves online at an accelerated pace. To fulfill their potential, given the changing operational context, archives have proactively introduced new cultural programming and supported it with fitting communication strategies: social media, live QQ\&A sessions, etc. We have observed more impact assessments being run, including user surveys. More archives are making strategic self-assessments of how their actions impact and offer value to society at large. This will lead to a deeper and more meaningful exploitation of the collections by a dedicated community of users. Fostering more direct engagement and reflecting on the audiences' expectations more intensively will be among the key factors determining the sector's recovery from the crisis.

Figure 4: FIAT/IFTA Timeline survey - alignment with specific user requirements 
$\infty$

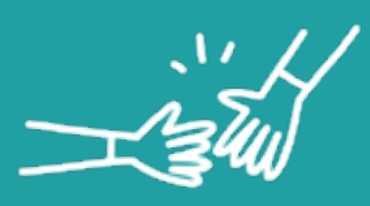

observation 3:

Explore

opportunities that new collaborations bring

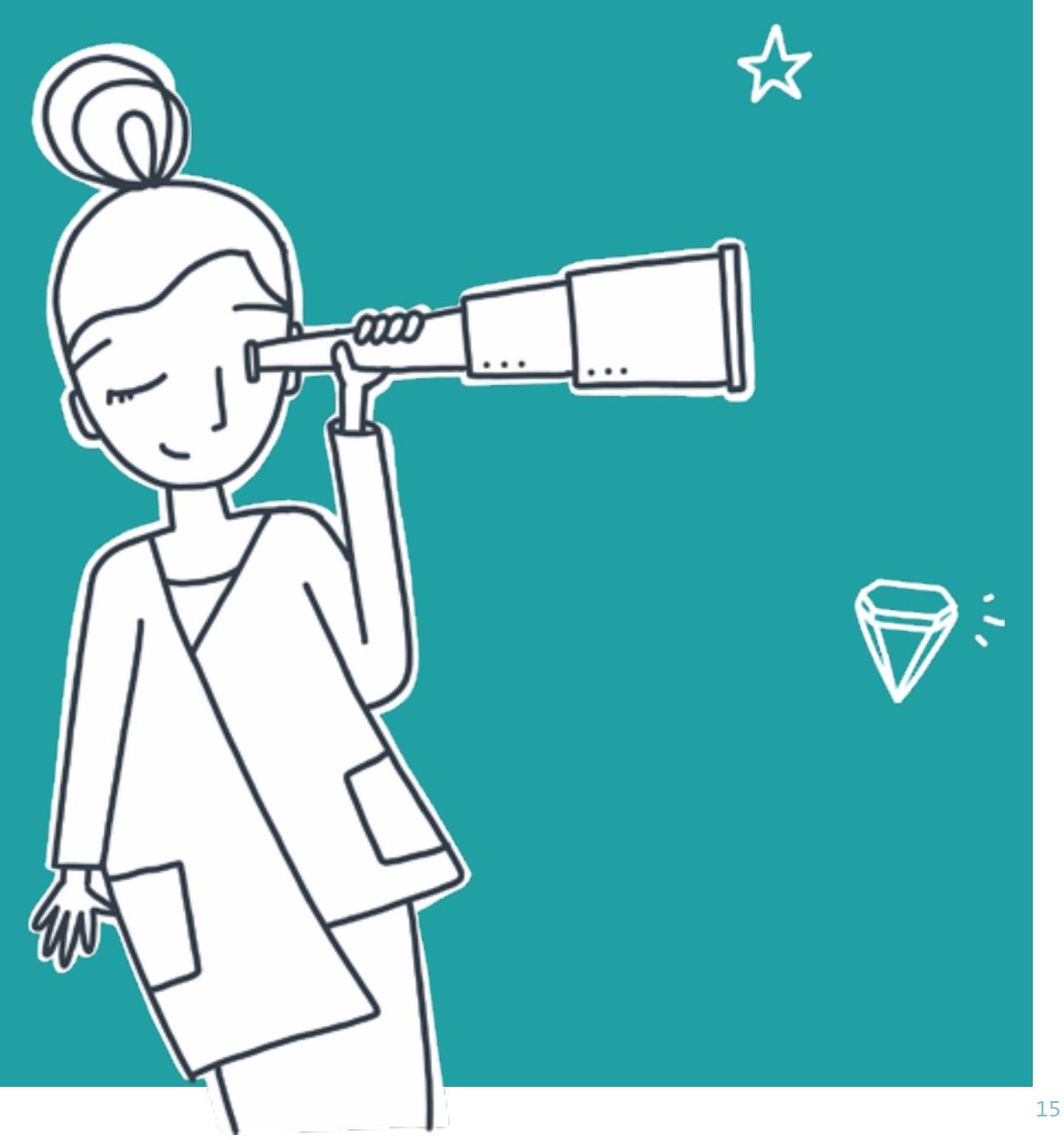


Now more than ever, archives should seek to ignite new partnerships (see Figure 5). Collections are relevant to many stakeholders, ranging from professionals in the creative industries looking to reuse material, to educational professionals, research communities, journalists, and the general public. In order to make best use of capacity and resources, partnerships are key.

Many participants mentioned the considerable growth potential of the educational market. Some archives began expanding their offering directly to this market, while others instead chose to partner with educational publishers and platforms such as massive open online courses (MOOCs), and use these existing channels to maximise their impact.

Another area of growing importance in the audiovisual domain is the effort to create a more healthy news ecosystem. Business and technology-oriented companies that prioritise attention-grabbing 'clickbait' news over responsible journalism in the public interest are gaining prominence. This development gives rise to the spread of misinformation, as manifested, for example, in recent elections and the circulation of untruths about vaccines. Archives can expand their role as authoritative voices and support public values. First, they can become trustworthy sources of information and provide access to collections to fact checkers and journalists who use big data to tell stories. Second, they can contribute to increasing media literacy in society by offering training and workshops to the general public. Strengthening partnerships with fact checkers, news outlets, and the broader community of journalists can reinforce the position of archives and contribute to their missions to build resilient societies through better informed citizens.

Third, there is an enormous potential to collaborate with the performing arts. As many music, theatre, and opera companies and venues are gearing up to make their performances available online, new opportunities for archives

In light of the COVID-19 crises it is important

\section{to search for new tools of creative}

collaborations between

audiovisual archives

and cultural and creative

sectors, research

institutions, etc.

Figure 5: FIAT/IFTA Timeline

survey - partnerships are emerging. For example, adding recordings of related content to the online catalogue of performing arts can be a fantastic way to create richer and more contextualised experiences. As performing arts venues are experimenting with the form of their online services, now is the time to seek collaborations. In many countries, performing arts organizations are now at growing risk of losing their analogue audiovisual archives due to a lack of investment in digitisation and digital collection policies. Mindful of these difficulties, institutions could pursue collaborations across a number of areas, including digitization, distribution, and experimenting with innovative experience formats.

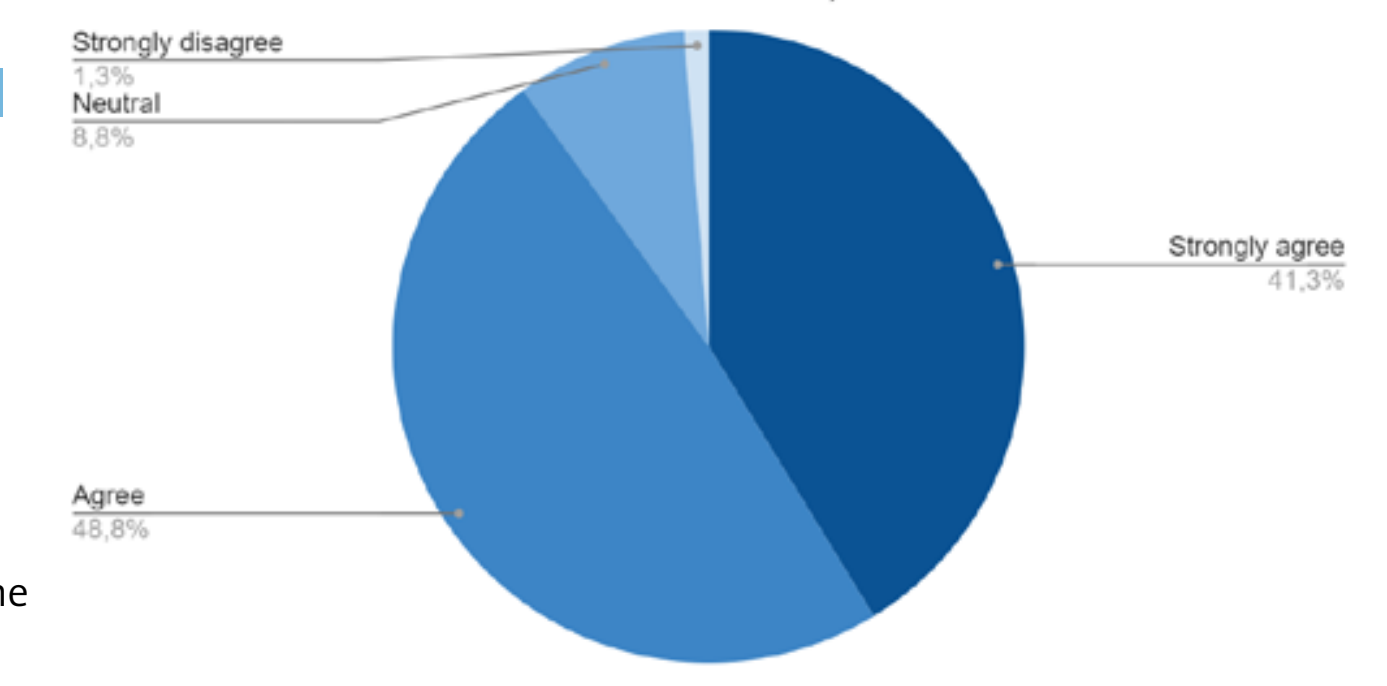


observation 4:
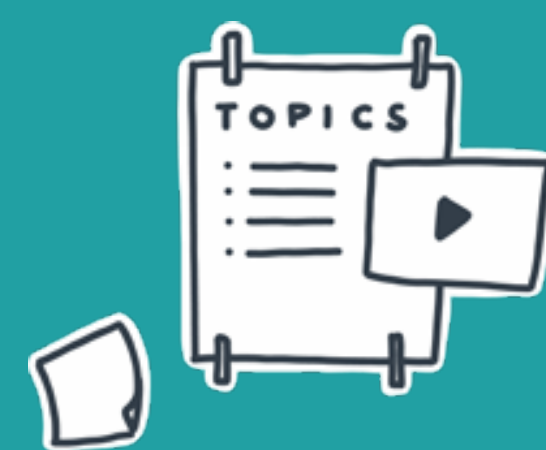

Redeffine how

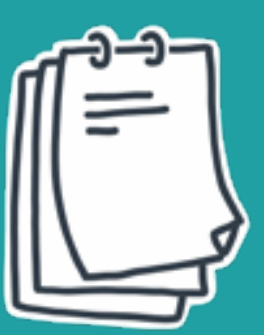

capacity building
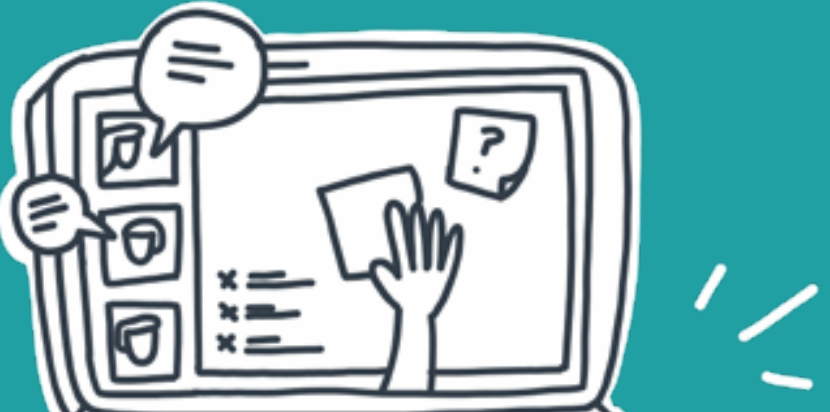

and knowledge

exchange takes shape 
No sector will grow and no change can be efficiently implemented without constant education and training to provide staff with new competences and qualifications. In early 2020 , this element of the archival profession was brutally disrupted. All professional events, conferences, seminars, workshops, internships, and other forms of knowledge exchange and training were suddenly postponed or cancelled. The organisers of the training courses and community events acknowledged that they had been taken by surprise. In the majority of cases, the adaptation to the new virtual realm took time. Global and regional stakeholders and individual archives offering training programs needed to develop possible scenarios for capacity building in a new environment, and were forced to devise a new approach towards in-person training - a must in some of the aspects of archival work.

Professional training and conferences offered by individual organisations, the professional sector, umbrella organisations, and training centres had to be adjusted to the new circumstances, making sure the professional community was able to follow the usual programs, while also developing the digital skills and new soft skills required for remote working to be fit-for-purpose (see Observation 5). The challenge was no longer simply to ensure the content quality of the learning programs. Many of us have gone through a crash course in moving capacity building initiatives online.

One year later, we know more. For instance, we acknowledge that some of the meetings and training can be easily moved online (e.g. conferences focusing on presentations, lectures, even interactive sessions with team collaboration). Others cannot, as nothing will replace a brainstorming and knowledge exchange session with a strong networking component held in a physical space. Direct interactions inspire us; they are thought-provoking, and very often lead to new professional connections and, in turn, collaborations. They are also the strongest incentive leading to community building. In this context, extra attention needs to be given to inviting young professionals entering the field, who need to build networks and gain hands-on experience. Examples of such efforts include reaching out to universities to create awareness of training and networking opportunities, and tailoring events to explicitly welcome and provide guidance to new community members.

Learning and teaching online means following new kinds of rules. Best practices show that online sessions should take no more than a few hours. In the past months, we observed conferences being spread out over multiple days and presenters being invited to pre-record their (shorter, rather than longer) presentations. That way participants could watch the contributions at their leisure, and the event itself could be focused fully on engagement. The online environment made conferencing more inclusive by removing the obstacles once posed by location, time, and resources, etc.

Undoubtedly, a whole new and vast range of capacity-building formats and tools is in place - in an online, hybrid, or on-site mode. While in-person training provides more hands-on experience, greater face-to-face interaction, and better networking opportunities, the online setting offers greater flexibility, can be more easily tailored to individual needs, and can be more inclusive. The opportunities are there, and the biggest current challenge is to properly balance the formats and methods of implementation, and to make sure they are fit to serve the needs of the community.

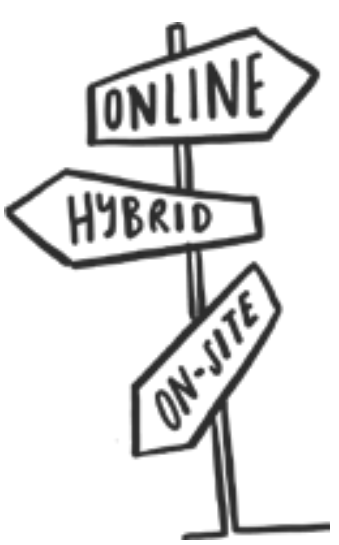




\section{observation 5:}

Invest to ensure working from home is fit for purpose

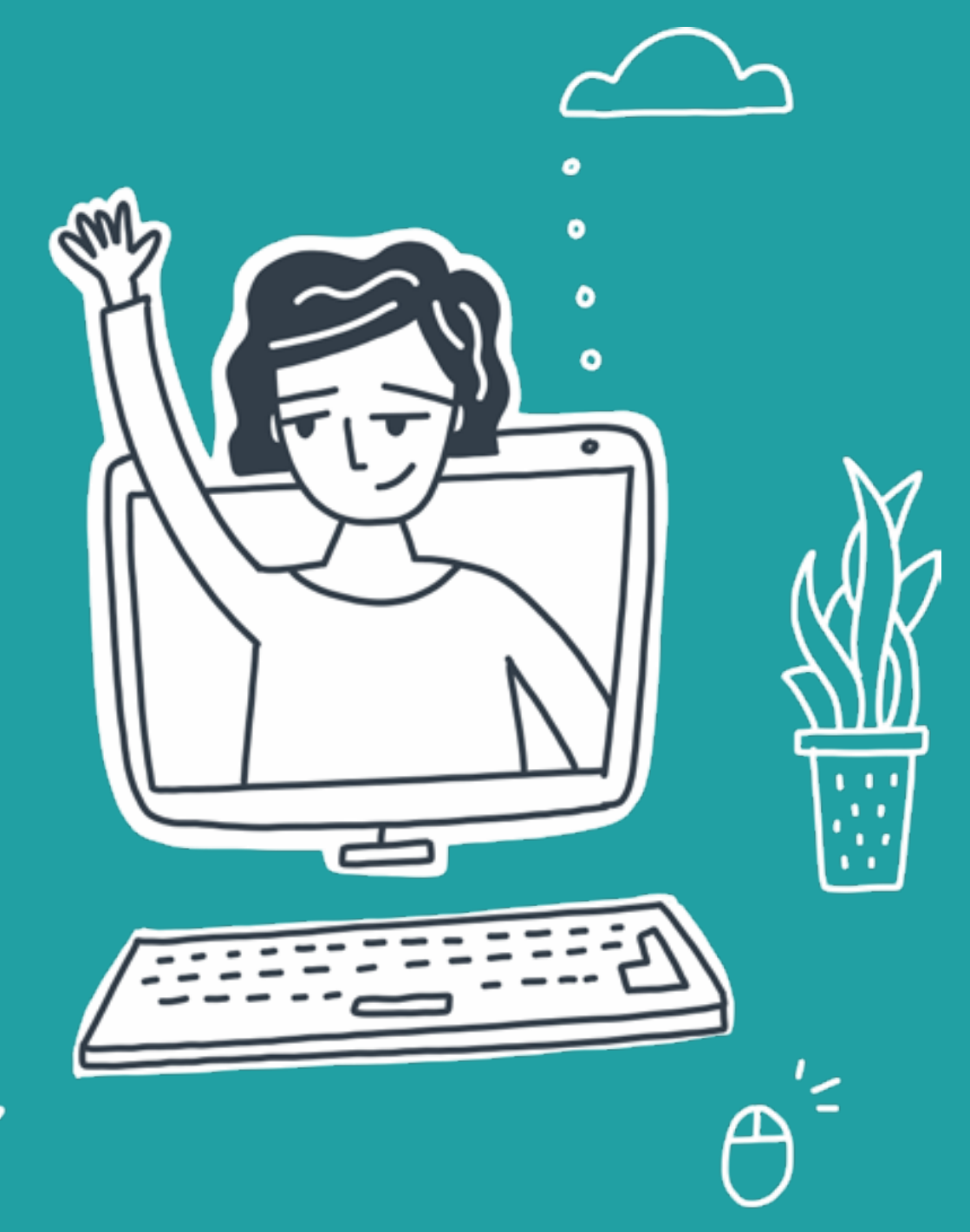


The past twelve months have witnessed drastic change in our working conditions. The community was forced to rapidly adjust its regular activities and workflows. It is expected that working remotely, in one form or another, is here to stay.

One resource that was deemed particularly useful in this context was 'Archivists at Home', a working document by the Society of American Archivists that addresses the needs of the archival community grappling with COVID-19. The living document contains a section titled 'Things archivists can do from home,', which subdivides several categories of work that can be done from anywhere. Some of the ideas shared are also relevant to audiovisual archives, the most obvious being creating and editing metadata. But other examples are mentioned, too:

- Crowdsourcing projects: e.g., create/update Wikipedia pages with relevant information from your institution's records;

- Administrative work: e.g., review rights statuses of collections and works published by your institution and held by other repositories/entities;

- Collection management: e.g., quality control review of digitized content or designing a virtual tour of your archives/collections/facilities;
- Collection development: e.g., compare the collection development policies and existing collections, plan out the steps for copyright clearance;

- Linked Data: explore ways to share your collections in aggregators/linked data;

- Reference/Outreach: create FAOs or 'Ready Reference' answers, podcasts, and video tutorials.

For many archives, the priority was to ensure staff and employees had the hardware they needed to work remotely. It was key that the team remained connected and, at least to some extent, was able to deliver the work. Some people mentioned archives facing new ways of working, including smart working, i.e., using new technologies and developing existing ones to improve both performance and job satisfaction. We see more and more of that trend around, with employees supported by an increasing number of tools and technical solutions.

Working from home has never been only about furnishing employees with the right futureoriented technical infrastructure. It's also about providing guidance on creating a comfortable, safe, and ergonomic home office. It's about time management and the ability to find the right productive mindset that will allow you to complete your daily tasks.
It is also about human connections. Stress, isolation, and the lack of direct contact with your team may lead to misunderstandings, conflicts, and even burnouts. Therefore it is crucial for teams working in the digital space to learn how to manage stress how to identify and mitigate it within the whole group by cultivating an environment and communication style that connects employees to the bigger picture and makes them feel like they're part of a team. The everyday relationships and interactions we have with people in our communities are fundamental, and thus need to be built on a values-based approach that foster kindness and partnership.

Last, but certainly not least, the challenge is also about considering the mental health of colleagues, especially those in more vulnerable categories (for instance, people with health conditions and those living alone), and making sure they retain the feeling of being part of the team and the culture of the organisation.

It is highly likely that we will continue to work more from home in the future. In order to ensure a smooth transition, archives need to define new organisational cultures, or redefine existing ones, acknowledging the working-from-home scenario and making the most of it. 
observation 6:

Become

an attentive

and agile

organisation

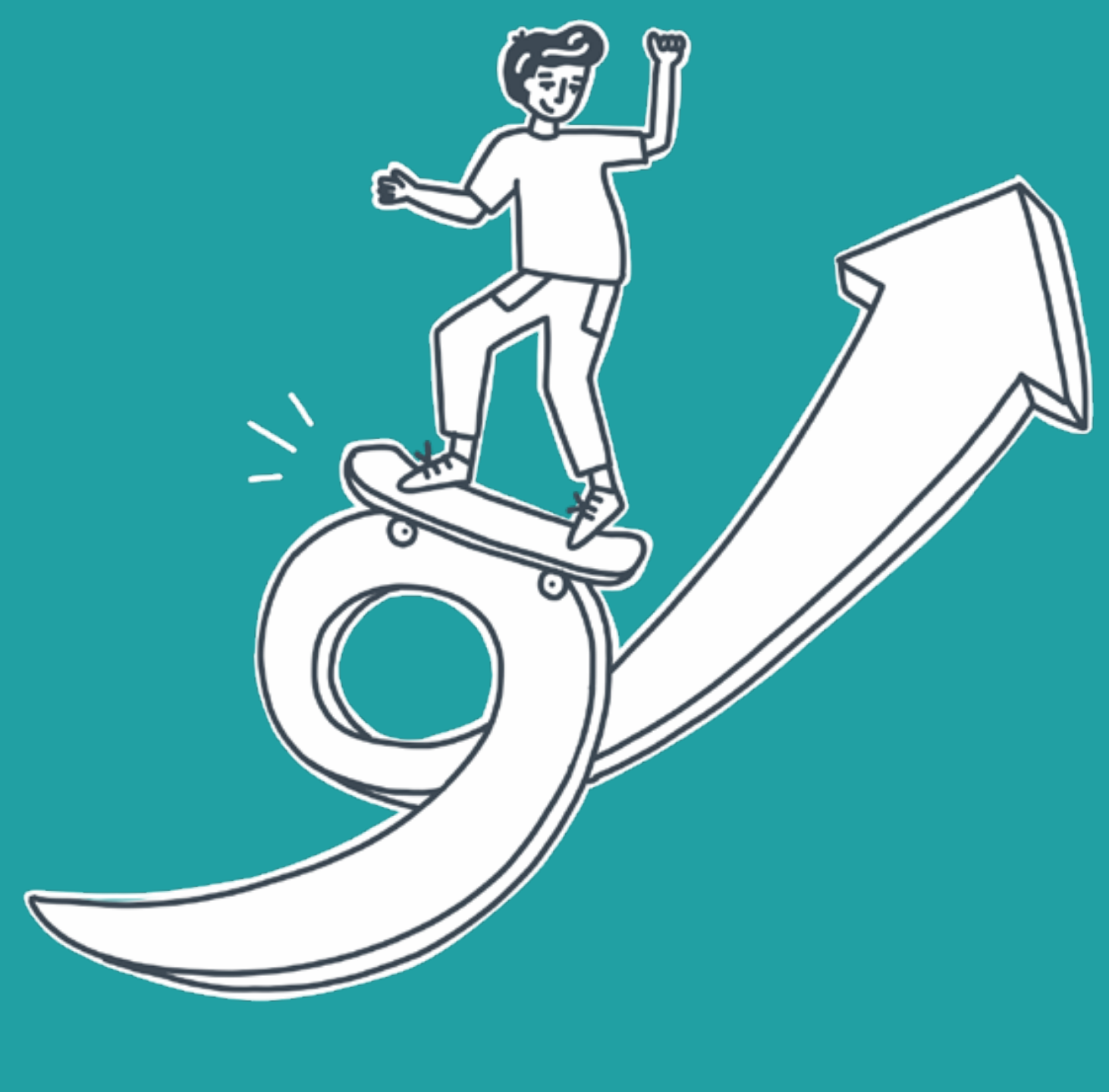


Archives developed a positive attitude about at least some aspects of remote working. Employees became familiar with using new digital tools (such as videoconferencing, virtual machines, and VPNs), and adjusted to the rhythm of working from home. With more flexible working hours and less time spent on commuting, the balance between work and social life has changed. Retaining a sense of culture within the organisation is important. Participants mentioned several inventive ways to relax with colleagues and chat about their experiences dealing with the lockdown. Examples included playing online games, karaoke, and even hosting remote disco parties. At the same time, flexible working hours at home may produce an 'always-on' effect. Finding the sweet spot between flexible working and a good work/life balance will be immensely important.

But there's more to the story of agility. Institutions participating in the virtual exchange shared examples of how organising work within their organisations and between teams became more agile. In one example, an internal 'job market' was set up where colleagues could share pickup work that needed to be done in other departments. This has proved to be a great way not only to make sure meaningful work is completed (and layoffs are avoided), but also to share knowledge across an organisation.

The need for more agility isn't exclusive to dividing work and organising remote working. For instance, it was noted that curation also required adaptation. For example, broadcasters were looking for more 'light' content from archives to provide much-needed distraction and entertainment. In many cases, curators/ archivists were consulted to help in the selection process. A more proactive position on the part of archives in the production and distribution chain will certainly help to increase visibility and reuse of collections. Therefore, institutions should also consider a 'rapid response' curatorial approach in the future.

The pandemic highlighted and exacerbated existing challenges related to social inclusion, engagement, and social inequality. In response, it was acknowledged that agility and attentiveness should be anchored in institutional policy, and that archives have a role to play that goes well beyond providing access to collections. Archives have repeatedly demonstrated their power to bring people together. Collections can be offered to support learning, knowledge sharing, to spark the recollection of memories, and for entertainment. This power of this public role of archives cannot be overstated. Now it is up to audiovisual archives, especially those funded directly by the public, to craft and execute institutional policies that will have a direct impact on society.

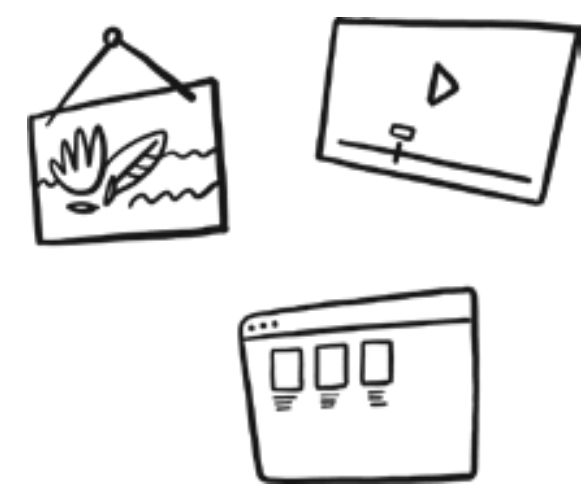

\section{Observations in a nutshell}




\section{COVID-19 AS A DRIVER FOR CHANGE IN AUDIOVISUAL ARCHIVES}

2

Foster direct engagement with your audience

1

Galvanise the full potential of online access to AV cultural content

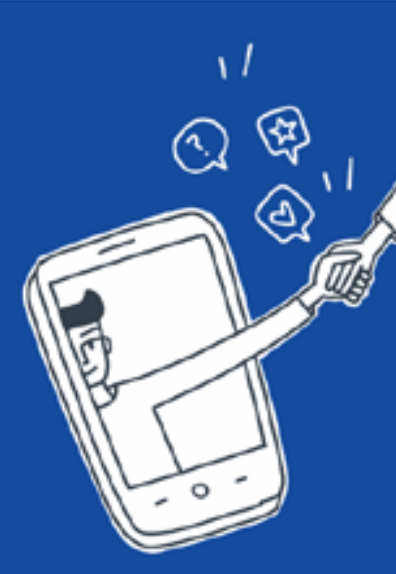

3 Explore opportunities that new collaborations is bring

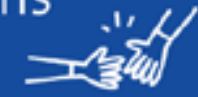

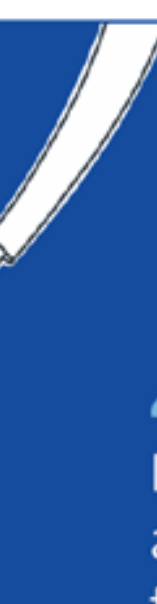

4

Redefine how capacity building and knowledge exchange takes shape
INITIAL OBSERVATIONS FROM THE VIRTUAL EXCHANGE BETWEEN AUDIOVISUAL PROFESSIONALS

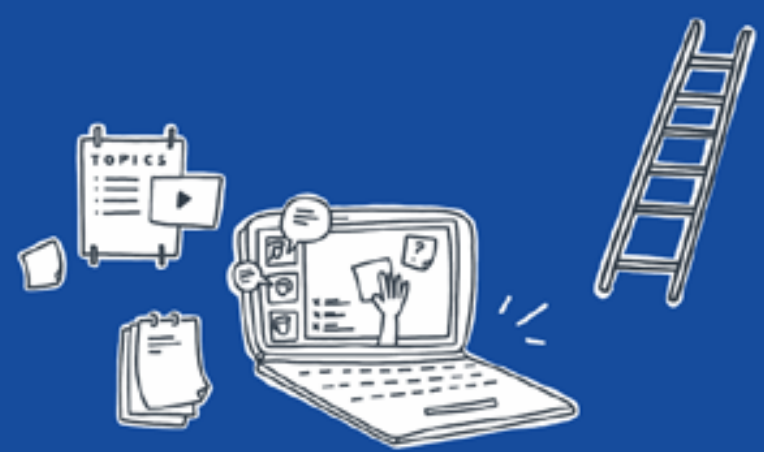

Invest to ensure working from home is fit for purpose
6

Become an attentive and agile organisation
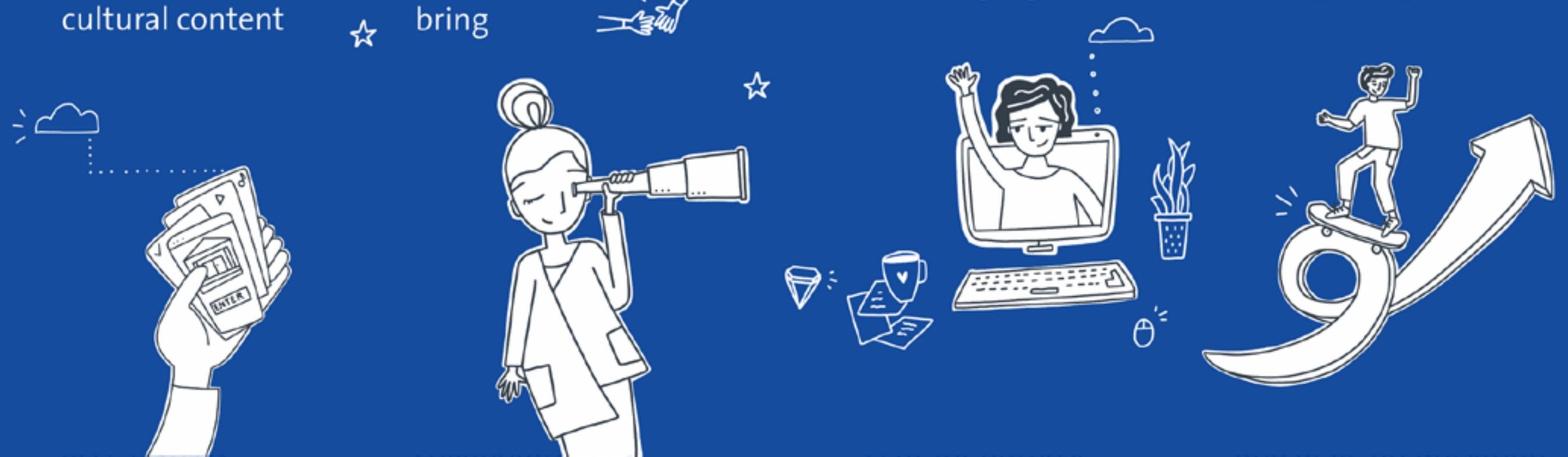


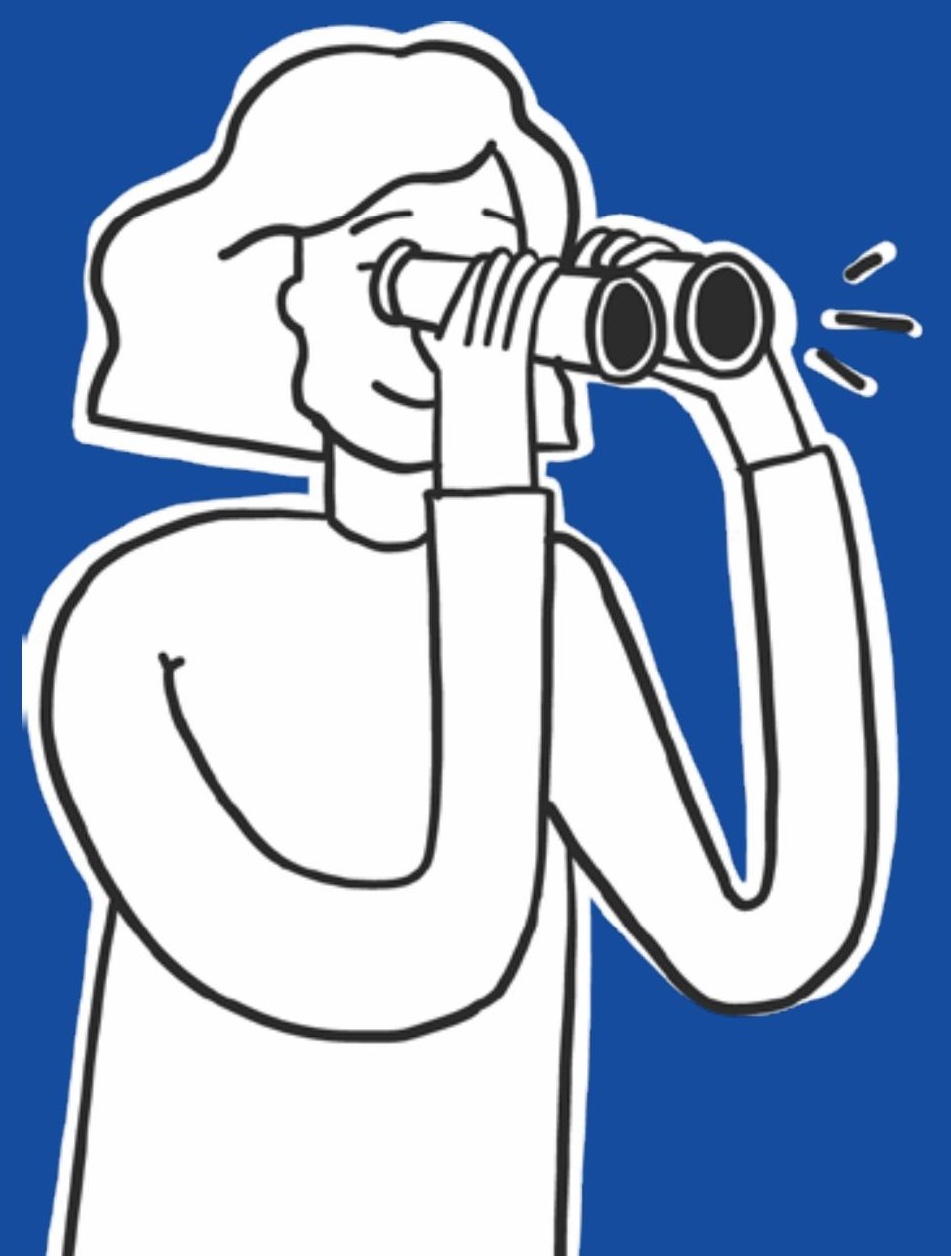


From the exchanges we have had over the past months, and from studies conducted by others, it has become apparent that we will emerge from this crisis stronger if archives innovate together and adopt future-oriented skills and strategies.

Archives, artists and others in the cultural and creative industries have a role to play to contribute to social cohesion. Bringing people together, supporting intercultural dialogue, and imagination will all be crucial to the successful implementation of sustainable development goals.

The observations presented in this document can be used as a source of inspiration an opportunity for online engagement and for exploring the potential to forge new collaborations. Notably, investments need to be made in digital technology and skills development to transform how organisations operate and deliver value.

The above discussion is part of a larger exchange among peers in the field. We encourage everyone to contribute by reaching out to the people involved in this study, or by following the social media channels of the umbrella organisations supporting this work.

https://twitter.com/fiat_ifta

https://twitter.com/iasa_web 